\title{
Covert video surveillance and the principle of double effect: a response to criticism
}

\author{
Elliot A Shinebourne Royal Brompton Hospital, London
}

\begin{abstract}
In some young children brought by their parents for diagnosis of acute life-threatening events investigations suggested imposed apnoea as the cause rather than spontaneous occurrence. Covert video surveillance of the cot in which the baby was monitored allowed confirmation or rebuttal of this diagnosis. That parents were not informed of the video recording was essential for diagnosis and we assert ethically justifiable as the child was the patient to whom a predominant duty of care was owed. The procedure also avoids the risk of separation of child from parent on inadequate information.
\end{abstract}

An ethical analysis ought to start with the facts. In this issue of the fournal of Medical Ethics, Thomas (1) criticises the use of covert video surveillance (CVS) in the investigation of Munchausen's syndrome by proxy, and specifically the protocol for its use published by the Staffordshire Area Child Protection Committee (2). Before addressing Thomas's criticisms of the protocol it is of note that his introductory paragraph contains misrepresentations of fact, albeit by proxy. To quote the first sentence: 'The secret filming of parents visiting their children in hospital to try and secure evidence of ill-treatment and child abuse has been the subject of some criticism' (3). As Thomas by now should know it was not the parents who underwent video surveillance but the baby (4).

What are the facts? Some infants or young children were referred to hospital because of acute life-threatening events such as severe apnoeic or cyanotic episodes with or without loss of consciousness or convulsions. The parents brought the child to hospital voluntarily with a request that the medical team determine the cause of these episodes. If the child had not already been neurologically damaged nothing was found on physical examination or on routine investigations so monitoring was set up. This included an electrocardiogram (ECG), pulse

\section{Key words}

Covert Video Surveillance (CVS); principle of double effect; Munchausen's syndrome by proxy; imposed apnoea; suffocation. oximeter to measure oxygen concentrations in the blood, a compressible capsule (Graseby) to detect respiratory movements, a pneumotachograph to record airflow at the nose and sometimes an electroencephalogram (EEG). Some documented episodes could not be explained by spontaneous events in the baby, but one explanation for the findings was imposed apnoea or suffocation. This was the circumstance when covert video surveillance of the baby was added to the monitoring system. A camera above the cot allowed videorecording of the baby in the cot while monitoring took place. In no way was this spying on the parents; it was used to document the aetiology of potentially fatal events the baby. If these episodes did occur spontaneousl 6 despite suspicions they were induced, this was show and the diagnosis of imposed apnoea refuted; whereas if the baby was being suffocated this would likewise be proven unequivocally.

The technique was not first used in the Academic Department of Paediatrics, North Staffordshire Hospital but was developed by Southall and colleagues at the Brompton Hospital, London. The particular circumstances when it was felt appropriate and ethically justifiable to use covert video surveillance were published in 1987 (4). Contrary to Thomas's assumption that a local research ethics committee had not reviewed the protocol, the protocol was presented to the research ethics committee of the Brompton Hospital, where after extensive debate it was approved. Stressing again that an ethical analysis should start with facts, most of the criticism of CVS in a recent paper by Evans (5) in this journal are based on the same mistaken premise. Even in the recent and balanced editorial by Gillon (6) there is confusion as to whether or not the use of CVS had been reviewed by a research ethics committee - it had - and whether or not it was an established clinical practice - it was, even if some disagree with the technique. As Southall and Samuels note in their rebuttal of Evan's criticisms, there are already papers in the literature reporting the technique $(4,8)$.

When CVS was first used, because of the ethical issues involved an open multidisciplinary meeting was arranged with social services, lawyers, psychiatrists, psychotherapists, social workers, experts in 
medical ethics, hospital administrators, the police, and medical and nursing staff to debate the issues. An ethical analysis of the issues discussed was reported by Dunstan (9) in the IME Bulletin. Self-evidently the central ethical dilemma was whether it was right to use video surveillance of the baby without the parent's knowledge if there were strong grounds to believe that acute life-threatening episodes were due to imposed apnoea (suffocation) rather than to naturally occurring events such as sleep apnoea, intermittent upper airways obstruction, prolonged expiratory apnoea or seizure activity. All personnel involved in implementing CVS were aware of the ethical dilemma. The children's unit at the hospital normally regard care of the child as a joint enterprise between the parents and the medical and nursing staff. Open discussion between parents and all health care personnel was the rule, since the idea of withholding information concerning their child from the parents was repugnant. Nonetheless the primary duty of care was to the child, who was the patient, and it was felt that the best way to protect these particular children was to ascertain for certain what was the aetiology of the acute life-threatening events, so they could be treated or prevented. This is not the place to debate whether there may be alternative means that would be as effective but at the time, and subsequently, no evidence has been produced to indicate that there are.

When CVS was undertaken at the Brompton Hospital the video monitors were viewed continuously by two women police officers so that should an untoward event occur the nursing staff could be rapidly summoned to come to the child's aid. It might have been easier to anticipate possible suffocation had the whole of the cubicle where the child was monitored been under video surveillance. Such an extension of the technique, however, was felt to be ethically wrong as this could be construed as spying on the family and certainly might invade a mother's privacy. By keeping only the baby on the cot under surveillance it was considered appropriate by the research ethics committee and by all personnel involved to be an acceptable procedure, even if there were still moral concerns about the action. In this decision we were mindful of the principle of double effect, understood as the situation when 'an action definable as good in terms of its object, can achieve a good effect only at the risk or expense of causing incidental but unavoidable harm (11).

In addressing more detailed comments by Thomas concerning the document published by the Staffordshire ACPC, I shall not deal with textual details so much as his judgment as to the implications of the text.

1) I agree with the statement 'when the evidence suggests a child is at risk the balance tips in favour of care for the child' although the language of rights then used is not helpful. Any one group of people's rights may be in conflict with another's rights and it is preferable to discuss the issues in terms of the duties of each group (which confer rights) as well as the harms which may be done to each group. For the child there is a duty of care from the health care professionals concerned, as well as from the parents. If the latter may have abused this duty of care then the primary duty of the health care professionals is first to protect the child.

What possible harm is done to the parent? If a parent is a perpetrator and is caught on video then the diagnosis is certain and appropriate action can be taken to protect the child and treat the parent. With this in mind it is noteworthy that in the 14 cases later published by Samuels et al (8) the 12 mothers concerned received non-custodial sentences and psychiatric care as did one grandmother - although the one father involved received life imprisonment.

If the parent is not the perpetrator and spontaneous onset apnoeic or cyanotic episodes are recorded on video the diagnosis is confirmed, the parent exonerated and appropriate treatment can be instituted. The parent would have been deceived or more explicitly not have been given full information prior to video recording. The harm to her or him is acknowledged but consists essentially of suspicions which turned out to be erroneous. Covert video surveillance (CVS) did not result in harm to the child, and most importantly did not result io removal of the child from an innocent parent.

Thomas writes later: 'If sufficiently strong evidence exists to justify the use of CVS it might be argued that it also exists to tip the balance in favour of action without recourse to CVS'. But what action? If trial separation of child from mother is in mind and the mother is not the perpetrator, I can think of nothing more cruel and harmful for the child and the mother. Thomas refers to the Cleveland Report where one of the major harms it was hoped to prevent was precisely this, taking the child away from the parents on inadequate evidence.

2) The agency receiving the referral will have already initiated a strategy discussion.

3) The appropriateness of CVS is of course first considered at the hospital to which the child is initially referred. Thomas fails to distinguish descriptive from prescriptive terms. As a matter of observation certain behaviour patterns were common in mothers who were suffocating their children.

4) Although Thomas seems prepared to accept that it is the child not the parent who is the patient of the paediatric team, he writes, 'alternatively it might be argued the real patient actually is the adult carer concerned even though he or she has not presented for treatment (1). Does this mean that the apnoeic child requiring resuscitation is not a real patient? Presumably Thomas does not mean that, but perhaps he envisages a competition between parent and child where only one of them is allowed patient status, the other being assigned the status of victim. 
5) Covert video surveillance (CVS) is a diagnostic activity that causes no harm to the child and may well prevent harm.

In circumstances where the diagnosis is uncertain a multidisciplinary meeting is held to discuss how best to make the diagnosis. It seems reasonable that this should be held before a formal meeting with parents, when inviting the latter may prevent the diagnosis being made. Even if care proceedings are already in process but there is no certainty that suffocation is the cause of acute life-threatening episodes, the use of CVS may prove that parents are not abusing their child. Thus if an episode caught on video is seen to commence spontaneously, the parent would be proven not to be the perpetrator of that event. It is the case, as Thomas reports, that an unattributed Lancet editorial (10) considered CVS to consist of vindictiveness under these circumstances but a contrary view is that it might help a parent refute allegations.

If a parent does decide to remove a child from hospital there is indeed a dilemma, but the parent may make this decision whether or not CVS is used.

I would agree that it is preferable that the police monitor the video surveillance rather than nurses. This point was also made by Dunstan, who stated that video surveillance is a proper activity of the police force and an activity for which they are trained, rather than for nurses whose role it is not (9). The problem in North Staffordshire, however, was that unlike their colleagues in London, the police in North Staffordshire would not assist CVS at the hospital (7).

The information obtained on video-tape recordings, especially if positive, is not of the same nature as child interviews. It is of note that when tapes have been viewed by nurses, doctors, other health care professionals or for that matter by Members of Parliament concerned about the ethics of CVS they have been deeply shocked by what they have seen, and the need to protect the child from such assaults in this way has been accepted.

6) Thomas is here concerned with what happens if the team gets it wrong and fails to intervene in time. Who would be responsible in any action for negligence? His primary concern now seems to be with the personnel undertaking CVS rather than with the child, an attitude that could be described as the 'out of sight, out of mind' approach to child abuse.

9) It may well be appropriate to inform parents that CVS was undertaken if an episode is recorded that occurs spontaneously and a positive diagnosis made. It may not be in the child's interest to tell the parents that CVS was undertaken if there were no episodes during the recording period as imposed apnoea or suffocation may still be the cause.
In Thomas's conclusion section there is a quotation from the Department of Health's current guidance on the Children's Act 1989 relating to child protection which promotes ... as much openness and honesty as possible between families and professionals.

There is no disagreement with this, but the aim is as much openness and honesty as possible. Sometimes it may not be possible to be open and to protect the child, so that difficult ethical dilemmas arise. Those who are not actively involved in protecting such children should perhaps be more reticent about criticising the actions of those who are endeavouring to do the best possible for the child even if that means making difficult ethical decisions.

Dr Elliot A Shinebourne, MD, FRCP, is Consultant Paediatric Cardiologist at the Royal Brompton Hospital, London.

\section{References}

(1) Thomas T. Covert video surveillance - an assessment of the Staffordshire Protocol. Fournal of medical ethics 1996; 22: 22-25.

(2) Staffordshire ACPC. Guidelines for the multi-agency management of patients suspected or at risk of suffering from life threatening abuse resulting in cyanotic-apnoeic episodes. Jan 1994.

(3) Foreman D M, Farsides C. Ethical use of covert videoing techniques in detecting Munchausen syndrome by proxy. Lancet 1993; 307: 611 .

(4) Southall D P, Stebbens V A, Rees S V, Lang M H, Warner J O, Shinebourne E A. Apnoeic episodes induced by smothering: two cases identified by covert video surveillance. British medical journal 1987; 294: 1637-1641.

(5) Evans D. The investigation of life-threatening child abuse and Munchausen's syndrome by proxy. Fourmal of medical ethics 1995; 21 : 9-13.

(6) Gillon R. Covert video surveillance by doctors for lifethreatening Munchausen's syndrome by proxy. Fournal of medical ethics 1995; 21: 131-132.

(7) Southall D P, Samuels M P. Some ethical issues surrounding covert video surveillance - a response. fournal of medical ethics 1995; 21: 104-105, 115.

(8) Samuels M P, McCloughlin W, Jacobsen R R, Poets C F, Southall D P. Fourteen cases of imposed upper airway obstruction. Archives of disease in childhood 1992; 67: 162-170.

(9) Dunstan G. The ethics of covert surveillance. IME bulletin 1988; 42: 15-17.

(10) Anonymous. Spying on mothers [editorial]. Lancet 1994; 343: 1373.

(11) Duncan A S, Dunstan G R, Welbourn R B. Dictionary of medical ethics [2nd ed]. London: Darton, Longmann and Todd, 1981: 145. 